\title{
Interactive Installations: Tales from the Trenches
}

\author{
Pedro Campos ${ }^{1,2}$, Miguel Campos ${ }^{3}$, and Joaquim A. Jorge ${ }^{2}$ \\ ${ }^{1}$ University of Madeira, Campus Universitário da Penteada, 9000-390 Funchal, Portugal \\ ${ }^{2}$ VIMMI Group, INESC-ID Lisbon, R. Alves Redol, 9, 1000-029 Lisbon, Portugal \\ ${ }^{3}$ WowSystems, Lda., R. Mary Jane Wilson, 21 G, 9050-446 Funchal, Portugal \\ pcampos@uma.pt, miguel.campos@wowsystems.pt, jaj@inesc-id.pt
}

\begin{abstract}
Breakthrough innovation can be interpreted as research translated into products that the market accepts. The process of market translation of several products developed by WowSystems, a Portuguese company specialized in novel interaction paradigms, is explained in this paper as a case study shedding some light into how innovation centers can better promote innovation, in the form of well-succeeded products. We describe two paradigmatic "tales from the trenches" and conclude with some guidelines that were outlined on the basis of more than three years delivering fifty interactive installations.
\end{abstract}

Keywords: Interactive Installations, Guidelines, Case Study, User Interfaces.

\section{Introduction}

Designing interactive installations for diverse venues and different contexts has become increasingly popular [1]. Science centers wish to exploit the interactive, often surprising, element to bring more visitors and to explain difficult scientific concepts in a more appealing way. Museums wish to attract visitors from all ages and promote collaborations between them. Retail stores and shops have also started to embrace interactive installations as a way to improve their relationship with existing clients as well as capture the attention and interest of new segments, exploiting installations featuring the so-called "wow!" effect. Moreover, the speedy evolution in computing power available, as well as the decreasing cost in display technologies, such as projectors and LCD displays, has also led to an increased level of interest from retailers wishing to improve their stores' attractiveness, museum curators wishing they had a nicer way to display the rich-ness of cultural heritage, science centers' managers who are simply technology enthusiasts and thrive with the idea of refurnishing their centers with the latest innovations. And the list goes on and on. Success seems almost certain in such a context. But sometimes the client says the project fails to deliver the intended effect. So, what went wrong?

This question is timely and, therefore, very relevant: what risks and opportunities do designers and developers face when delivering real world innovative interactive installations? By defining breakthrough innovation as research prototypes translated into products that the market accepts, we describe our own experience in bridging research prototypes developed at the University of Madeira and at INESC-ID Lisbon, and studying how they were translated into real world applications in different 
contexts. We have been lucky enough to work around several practitioners' issues and risky situations in this field, and we have been working towards compiling sets of guidelines based on both successful and not so successful projects. While some of the more than fifty interactive installations already deployed were solely created as experiential activities, providing an increase in the level of learning by adding facts to an already well-formed conceptual model, others were designed to enact a reflective activity, thus supporting a restructuring learning where new conceptual frameworks need to be built. Based on this experience, we have summarized into a set of guidelines some ways to help interaction designers survive and perform well when the expectations are increasingly getting higher.

\section{From the Interactive Installations' Trenches}

It has been argued that the identification of breakthrough ideas at the very forefront of the innovation process is a key factor towards the creation of substantial innovation [2]. However, the managerial process of breakthrough innovations, as well as their inhibitive factors, remains far from being understood [2].

In this paper, we briefly describe our own experience with WowSystems, a Portuguese company specialized in new digital media, novel interaction paradigms and interactive installations. Because of the very nature of its core business, innovation is - and should continue to be - a main concern of the company. Founded in early 2008, WowSystems' main focus has been to professionally create useful and usable interfaces that make people go "Wow!".

Two tales from the trenches we selected are a cultural interactive exhibition and an interactive shoe store. WowSystems designed a set of sensor-based installations in a cultural exhibition organized by the Direction of Cultural Affairs, which aimed at showing the visitor the cultural richness that formed the streets of Funchal (Portugal). The concepts of the exhibition revolved around promoting awareness about, and foster a better understanding of, the cultural tourism that can be performed by simply walking through strategic streets and watching certain buildings, sites, and heritage. To better complement the exhibition's traditional large-format printed panels, the organizers wanted to have the interactive factor as a means to add value to the visitor's experience. The left photo in Fig. 1 illustrates an installation in this exhibition.

In a similar project, we designed and installed and interactive mirror for a shoe shop, illustrated in the right photo in Fig. 1. The client's expectations included: That the shoe shoppers would step inside an RFID-tagged shoe and watch themselves inserted into a real time virtual scenery related to the type of shoe they were trying on. Our design had the following characteristics: As a shopper walks around the experimenting floor, the shoe's RFID tag is read by the reader, then the model's attributes are fetched from the product database, sent to the multimedia server which displays two synchronized scenarios: one for two top-down projections (left photo) and one for the front, "mirror-like" view (right photo).

The "mirror-like" front view displays the shopper in real time and places her on a virtual scenery by using a motion detection and silhouette extraction algorithm. This algorithm is adaptive regarding the different lighting conditions at the shop - usually brighter during the day and darker at dusk and night. Top-down projections feature 
views of the streets or sidewalks that are typical of the city that the virtual scenery replicates. For instance, the photo shows a shopper trying a shoe model that had a design inspired by modern life in Tokyo. Therefore, our interactive mirror displays a scenery based around Tokyo's neon signs and bright buildings. Simultaneously, the floor projections display a Tokyo sidewalk with Japanese signs and warnings, as well as other visual elements, and add interactivity by displaying neon lights over the floor according to the shopper's position.
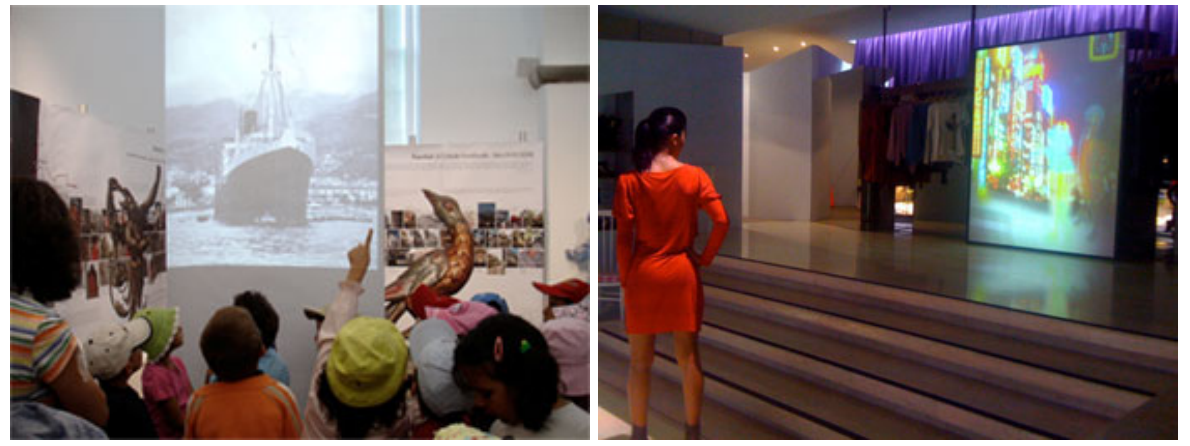

Fig. 1. Two examples taken from the trenches: an interactive cultural exhibition (left) and a virtual mirror for a shoe store

The first interactive exhibition project went very well and post-project analysis suggests that good communication with museum curators, artists and designers was crucial to its success. A good mapping of the interaction styles into the installations and the exhibitions' theme was also important to grab the visitors' attention.

Upon the final installation of the shoe store, however, the solution didn't fulfill the client's expectations. Post-project analysis suggests one of the reasons this happened was simply because the expectations were put too high. Contrarily to other projects delivered to this client by other companies, however, our solution fitted the consumers' profiles very well, and the high satisfaction levels that shoe shoppers expressed were crucial in helping us defend the project's solutions.

\section{Conclusions}

Because of today's diversity of possible technological combinations for any interactive installation, the solution space has become too large. This, we argue, is an issue that contributes to increasing risks in interactive installations' development. And it's one of the reasons why it is surprisingly easy to create bad designs.

Secondly, experience has shown that during an economic crisis some clients start focusing on finding excuses for not admitting a project's success-and therefore not paying. The problem with frontline interaction design is that it's fairly easy to debate or discuss the final results of an installation: People's tastes are highly subjective and vary a lot. Requirements engineering as a discipline has many principles, techniques, and methods devoted to traditional software development. However, in terms of validating interaction design requirements, research literature is somewhat scarce. More 
effort should be put into how we can more effectively work collaboratively with stakeholders in order to better define the interaction design aspects of any given project's requirements.

The guidelines we identify on the basis of our experience are our own way to deal with the difficulties, risks and opportunities that come up in this field. They could prove useful for other interaction designers, business managers, and even clients, as a way to design, develop and install better products. These guidelines include, but are not limited, to the following.

Making the Vision Stand Out. It is a good idea to hang exhibition posters that feature interactive installations, photos of the visitors, and, for instance, give away free tickets, whenever applicable, so that engineers and designers can experience the installations the exact same way clients and users do.

Know the customer from the client. Interactive installations are meant to be fun, enriching, and enticing to everyday customers. A successful installation will attract more customers and more business, therefore making your client happy. The focus should be on your client's customers and not on your clients. A good defense mechanism to support design decisions is to convincingly and accurately document the customers' satisfaction and deliver that documentation to your client with a partnership attitude. Collecting evidence such as happy customers' photos, videos of people interacting with the installations, even surveys or informal interviews, can be useful to convince your client, especially if cross-checked with sales or other business figures. Please your client's clients.

Carefully manage client expectations. One way to achieve this is to present the client with architectural designs of how the interactive installation will look at the end of the project. If we provide the client with a visual scale and 3-D layout, the idea can be conveyed in a way that gives all stakeholders a feel of how the physical space will be used for the installations, just like in architectural programs.

Acknowledgements. This work has been supported by FCT (the Portuguese Foundation for Science and Technology) through grant PTDC/EIA-EIA/116070/2009.

\section{References}

1. Liu, W., Fernando, O.N., Cheok, A.D., Wijesena, J.P., Tan, R.T.: Science Museum Mixed Reality Digital Media Exhibitions for Children. In: Proceedings of the Second Workshop on Digital Media and Its Application in Museum \& Heritage, December 10-12, pp. 389-394. IEEE Computer Society, Washington, DC (2007)

2. Funnel or tunnel? A tough journey for breakthrough innovations. In: Proceedings of the 4th IEEE International Conference on Management of Innovation and Technology, ICMIT, pp. 368-373 (2008) 